\title{
Microwave reflectometric systems and monitoring apparatus for diffused-sensing applications
}

\author{
Andrea Cataldo ${ }^{1}$, Egidio De Benedetto ${ }^{2}$, Raissa Schiavoni ${ }^{1}$, Annarita Tedesco ${ }^{1}$, Antonio Masciullo ${ }^{1}$, \\ Giuseppe Cannazza ${ }^{1}$ \\ ${ }^{1}$ Dept. of Engineering for Innovation, University of Salento, Lecce, Italy \\ 2 Dept. of Electrical Engineering and Information Technology, University of Naples Federico II, Naples, Italy
}

\begin{abstract}
Most sensing networks rely on punctual/local sensors; they thus lack the ability to spatially resolve the quantity to be monitored (e.g. a temperature or humidity profile) without relying on the deployment of numerous inline sensors. Currently, most quasi-distributed or distributed sensing technologies rely on the use of optical fibre systems. However, these are generally expensive, which limits their large-scale adoption. Recently, elongated sensing elements have been successfully used with time-domain reflectometry (TDR) to implement diffused monitoring solutions. The advantage of TDR is that it is a relatively low-cost technology, with adequate measurement accuracy and the potential to be customised to suit the specific needs of different application contexts in the 4.0 era. Based on these considerations, this paper addresses the design, implementation and experimental validation of a novel generation of elongated sensing element networks, which can be permanently installed in the systems that need to be monitored and used for obtaining the diffused profile of the quantity to be monitored. Three applications are considered as case studies: monitoring the irrigation process in agriculture, leak detection in underground pipes and the monitoring of building structures.
\end{abstract}

Section: RESEARCH PAPER

Keywords: Time-domain reflectometry; industry 4.0; microwave sensing; concrete monitoring; diffused monitoring; dielectric permittivity

Citation: Andrea Cataldo, Egidio De Benedetto, Raissa Schiavoni, Annarita Tedesco, Antonio Masciullo, Giuseppe Cannazza, Microwave reflectometric systems and monitoring apparatus for diffused-sensing applications, Acta IMEKO, vol. 10, no. 3, article 1, September 2021, identifier: IMEKO-ACTA10 (2021)-03-01

Section Editor: Section Editor

Received July 26, 2021; In final form September 1, 2021; Published September 2021

Copyright: This is an open-access article distributed under the terms of the Creative Commons Attribution 3.0 License, which permits unrestricted use, distribution, and reproduction in any medium, provided the original author and source are credited.

Funding: Italian Ministry of Universities and Research (MUR) through the project 'SMARTER-Systems and Monitoring Apparatus based on Reflectometric Techniques for Enhanced Revealing' (POC01-00118), funded under the Public Call Proof of Concept D.D. n. 467 of 02/03/2018.

Corresponding author: Egidio De Benedetto, e-mail: egidio.debenedetto@unina.it

\section{INTRODUCTION}

Monitoring systems represent a key enabler for the 4.0 era, crucial not only for guaranteeing the optimal functioning of the monitored systems but also for timely interventions in case of potential failures [1], [2]. As a result, the combination of the Internet of Things (IoT) and sensing networks has become an irreplaceable tool for achieving ubiquitous monitoring in the 4.0 ecosystem [3]-[8]. However, currently, most sensor or monitoring systems are characterised by point-type sensory information; hence, to monitor large areas, it is necessary to employ a multitude of probes. This drawback can be overcome by using diffused sensing elements (SEs or d-SEs) that are able to achieve many functions [10] where the use of point sensors is not recommended. Most SEs of this type rely on the use of optical fibre systems [11], [12], but optical systems are generally expensive, and this limits their large-scale adoption. In this paper, the diagnostic technology employed resides in time-domain reflectometry (TDR) through the use of elongated SEs, which have recently been successfully used to achieve diffused monitoring [13]-[21]. TDR monitoring systems are characterised by being relatively low cost, having the potential to be customised to suit the specific needs of different applications and achieving good accuracy. For these reasons, this technique represents an interesting monitoring solution with the use of specific sensing element networks (SENs) that can be permanently embedded into the system to be monitored (STBM) and used throughout the service life of the STBM. Thanks to the versatility of TDR, the proposed system can be customised and applied in a considerable number of fields; but this paper focuses on three contexts: 
i) localising leaks in underground pipelines (SEN-W),

ii) agricultural water management (SEN-A) for the optimisation of water resources,

iii) building monitoring (SEN-B) through the ex-ante monitoring of concrete curing and ex-post monitoring for the detection of dielectric anomalies that result from the degradation or stress of the structure.

The paper is organised as follows. Section 2 describes the basic theoretical background of TDR, while Section 3 describes the design and implementation of the proposed distributed monitoring system. In Section 4, the experimental results of the practical implementation of the proposed SENs are reported, and finally, in Section 5, conclusions are drawn, and the future work is outlined.

\section{THEORETICAL BACKGROUND}

TDR is an electromagnetic (EM) measurement technique typically used for monitoring purposes, such as food analysis [22], cable fault localisation [23]-[26], soil moisture measurements [27], liquid level measurements [28], device characterisation [29], [30], biomedical applications [31] and dielectric spectroscopy [20]. In TDR measurements, the EM stimulus is usually a step-like voltage signal that propagates along the SE, which is inserted or in contact with the system under test (SUT). The signal travels along the SE, and it is partially reflected by impedance changes along the line and/or by dielectric permittivity variations. Through the analysis of the reflected signal, it is possible to retrieve the desired information on the SUT. Generally, in TDR measurements, the direct measurement output is the time-domain reflection coefficient $(\rho)$, expressed as

$$
\rho=\frac{v_{\text {refl }}(t)}{v_{\text {inc }}(t)},
$$

where $v_{\text {refl }}(t)$ is the amplitude of the reflected signal and $v_{\text {inc }}(t)$ is the amplitude of the incident signal. The value of $\rho$ is represented as a reflectogram, which shows $\rho$ as a function of the travelled apparent distance $\left(d_{\mathrm{app}}\right)$. As is well known, the quantity $d_{\text {app }}$ is related to the actual distance, $d_{\text {real }}$, by the following equation:

$$
d_{\mathrm{app}}=d_{\mathrm{real}} \cdot \sqrt{\varepsilon_{\mathrm{app}}}=\frac{c \cdot t}{2} \sqrt{\varepsilon_{\mathrm{app}}},
$$

where $\varepsilon_{\text {app }}$ is the effective dielectric permittivity of the propagating medium (which describes the interaction between the electromagnetic signal and the SUT), $c$ is the velocity of light in free space and $t$ is the travel time, which is the time that it takes for the EM signal to travel back and forth.

The signal propagation velocity inside the medium depends on the dielectric properties of the material in terms of effective relative dielectric permittivity, $\varepsilon_{\text {app }}$, which describes the interaction between the electromagnetic signal and the SUT. If the EM signal is propagated in a vacuum, then $\varepsilon_{\text {app }} \cong \varepsilon_{r \text {,air }} \cong$ 1.

However, if the SE is inserted in a material different to air, then the propagating EM signal will propagate more slowly, and the effective dielectric constant of the material in which the SE is inserted through the estimation of the apparent length $\left(d_{\mathrm{app}}\right)$ is evaluated from the reflectogram:
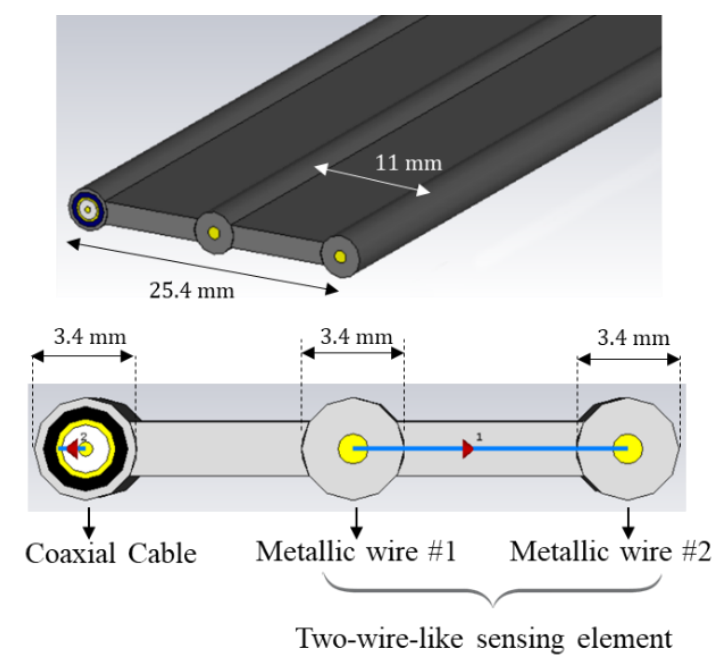

Figure 1. Designed diffuse sensing element: two-wire-like conductors and a coaxial cable.

$$
\varepsilon_{\mathrm{app}}=\left(\frac{d_{\mathrm{app}}}{d_{\mathrm{real}}}\right)^{2} \text {. }
$$

Based on these considerations, using the TDR reflectogram, it is possible to estimate the dielectric characteristics of the propagating medium and/or to localise when these dielectric variations occur.

\section{SYSTEM AND DESIGN IMPLEMENTATION}

\subsection{Diffused sensing element}

As mentioned in Section 1, the present study focuses on three main application fields. In detail, the SEN-W refers to a leak localisation system in underground water and sewer pipes, SEN$\mathrm{A}$ is dedicated to the real-time monitoring of the soil watercontent profile in agriculture and, finally, SEN-B addresses the use of the TDR and elongated SEs to evaluate the humidity profile of concrete structures and to identify destructive phenomena early, such as those resulting from rising damp. This section describes in detail the design and implementation of the d-SEs, the TDR measuring instruments used for experimental validation and the processing algorithm adopted. Before the implementation of the system, in the design phase, full-wave simulations were carried out to identify the optimal SE configuration, which is useful for optimising the performance of the system in terms of sensitivity to changes in the dielectric characteristics of the STBM. The configuration of the diffused $\mathrm{SE}$ is shown in Figure 1. It consists of a coaxial cable and two conductors that run parallel to each other and are mutually insulated through a plastic jacket. The figure also shows the cross-section dimensions of the SE. The sensing portion is placed along the direction of the electrical impedance profile under test so as to provide diffused monitoring of the STBM. The EM signal propagation occurs between the two conductors and is influenced by the dielectric characteristics of the surrounding material; this aspect is exploited to identify the area in the reflectogram in which dielectric variations are observed. The coaxial cable has the same length as the sensitive portion and is integrated with the SEs to calibrate the apparent distance as a real distance. In fact, because the dielectric characteristics of the coaxial cable are known, by propagating the TDR signal along the coaxial cable, it is possible to evaluate the actual distance of 


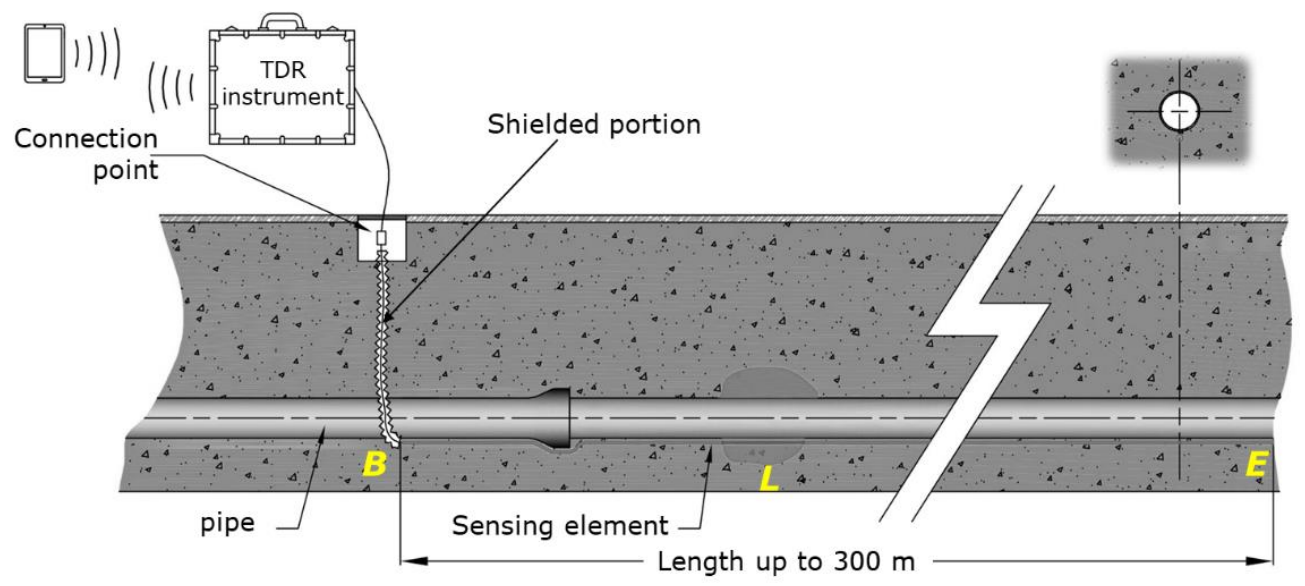

Figure 2. Schematisation of the TDR-based system for SEN-W (dimensions not to scale).

the SE from (3), as reported in [15]. This aspect is especially important in practical applications in which the length of the dSEs is not necessarily known in advance.

\subsection{TDR instruments and the measurement algorithm}

For the TDR measurements, two measuring instruments were used, the TDR-307USBm and the TDR200. The former generates a pulse signal, and the cost is relatively low (about $€ 800)$. The latter generates a step-like EM signal, and the cost is

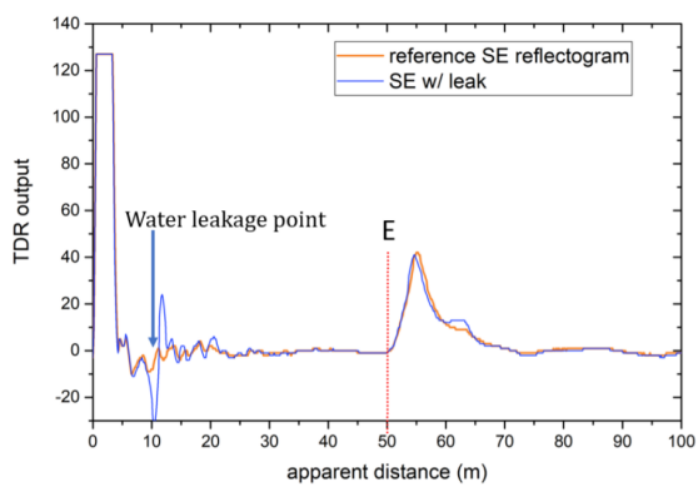

(a)

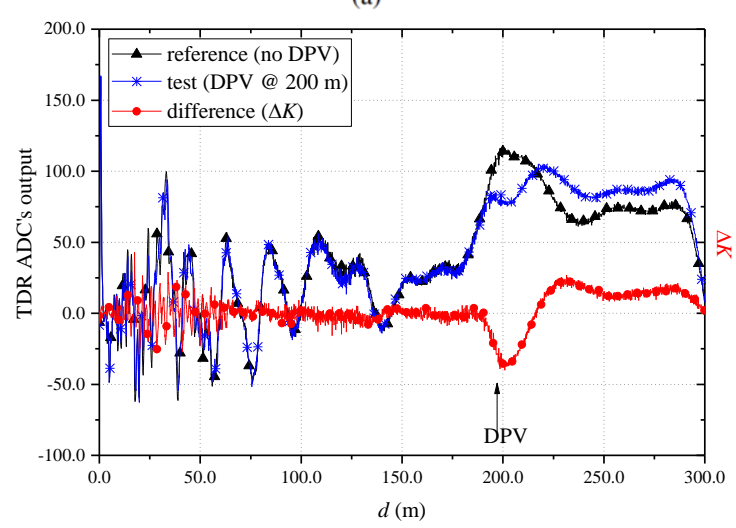

(b)

Figure 3. SEN-W: a) comparison of the test reflectogram and reference reflectogram for leak detection. b) Localisation of the dielectric permittivity variation (DPV): reference reflectogram is superimposed on the test reflectogram. The difference between the two reflectograms is also shown (red curve). approximately five times the cost of the TDR-307USBm. Both measuring instruments are portable.

However, in the case of the TDR-307USBm, it is worth mentioning that signal attenuation and dispersion phenomena limit the usability of TDR when used on long cable systems. It is possible to modify the amplitude and the width of the TDR test pulse signal along with the gain in the input stage amplifier; however, this requires the TDR measurements to be repeated many times to find the right setting.

To overcome this issue, an innovative and dedicated algorithm [16] has been developed to automatically optimise these two different electrical parameters of the TDR signal as a function of the d-SE length in order to compensate the performance in terms of attenuation and dispersion effects.

\section{EXPERIMENTAL RESULTS}

\subsection{Experimental results for SEN-W}

Water-pipeline monitoring is extremely important given the considerable amount of water loss caused by leakages and other possible hydraulic failures. Figure 2 shows a schematisation of the setup configuration. During the installation phase, the d-SE is placed along the pipeline to be inspected, and the connection to the measuring system is ensured through an access point. Points $B$ and $E$ indicate the beginning and the end of the d-SE, while $\mathrm{L}$ indicates the position of a leak. It can be seen that there is a section of cable (running vertically) that allows the SE to be connected to the measurement instrument.

Clearly, variations in dielectric permittivity that may occur along this vertical SE section are not of interest for the leak localisation. To overcome this issue, this portion of $\mathrm{SE}$ is electromagnetically shielded (by means of a metal shield). In this way, the sensing portion starts from point $B$.

Another advantage is that, thanks to this shielding, the vertical portion can be of any length, as it does not influence the localisation of the leak (hence, it is not necessary to know a priori the burial depth of the pipe).

This approach allows easier identification of the interface relative to the start point of the SE relative to the buried pipeline, excluding the portion of the connected section from the measured data.

For the experiments on SEN-W, a dedicated testbed was set up, in which a d-SE was buried approximately $30 \mathrm{~cm}$ 
underground. The soil was irrigated at predetermined distances from the beginning of the $\mathrm{SE}$ in a similar way to a leakage condition when water escapes from an underground pipe. The main parameter describing the interaction between the signal and the system under test is the relative permittivity $\varepsilon_{\mathrm{r}}$, as water leakage causes a local variation in its value, which is immediately detectable in the measurement. For these measurements, the TDR RI-307USBm was used. In fact, because SEs in these applications may be a hundred meters long, the TDR200 instrument is unsuitable, as its electronics could be easily damaged as a result of electrostatic discharges occurring when connecting long cables.

Figure 3(a) shows the measurement results obtained for a leak at $\mathrm{d}=8 \mathrm{~m}$. First, the reference reflectogram was acquired, i.e. when there is no leak (red curve). Then, the reflectogram in the presence of the emulated leak was acquired. It can be observed that, in the presence of the leak, there is a distinct variation in the output reflectogram. The position of the leak is estimated by applying (2) to the different portions of the reflectogram in order to identify the abscissa of the minimum corresponding to the leak. It is also interesting to analyse the detection of a leak (or a dielectric permittivity variation (DPV)) at long distances (i.e. 200 $\mathrm{m})$, which is when the use of the aforementioned algorithm becomes essential. Figure 3(b) shows the TDR curves that are automatically processed; the difference between the test reflectogram and the one in the presence of a DPV allows the DPV to be localised.

It should be mentioned that in applying (2), an assumption of constant permittivity in the soil along the SE is made. This assumption is acceptable considering that, generally, the variation in permittivity caused by leakages is significantly higher than the natural variation of permittivity in the soil (which may be due to temperature variations or to slightly different soil compaction in different areas).

\subsection{Experimental results for SEN-A}

In this application context, monitoring soil moisture content in agriculture allows the use of water to be optimised, reducing wastage. In particular, the basic idea is to bury the d-SEs in correspondence with cultivations and to carry out TDR measurements to retrieve the actual water-content profile of the soil all along the cultivations. Subsequently, the irrigation systems can be automatically activated/deactivated according to the actual irrigation needs of the plants. In addition, through multiplexing systems, up to $512 \mathrm{~d}$-SEs could be used simultaneously to perform the widespread monitoring of multiple crop rows. For the experimental tests, a 30-m-long d$\mathrm{SE}$ was placed in the soil along the entire crop profile, and the connector came out of the soil to connect to the TDR measurement instrument. In this case, for the sake of comparison, both the TDR200 and the TDR RI-307USBm were used. The acquired reflectograms were directly related to the impedance profile of the SE inserted in the soil. Experimental results were obtained according to the following measurement protocol:

- reflectogram \#1: one week after d-SE installation,

- reflectogram \#2: (post-irrigation) acquired approximately one hour after the irrigation process was finished,

- reflectogram \#3: (pre-irrigation) acquired three days after reflectogram \#2,

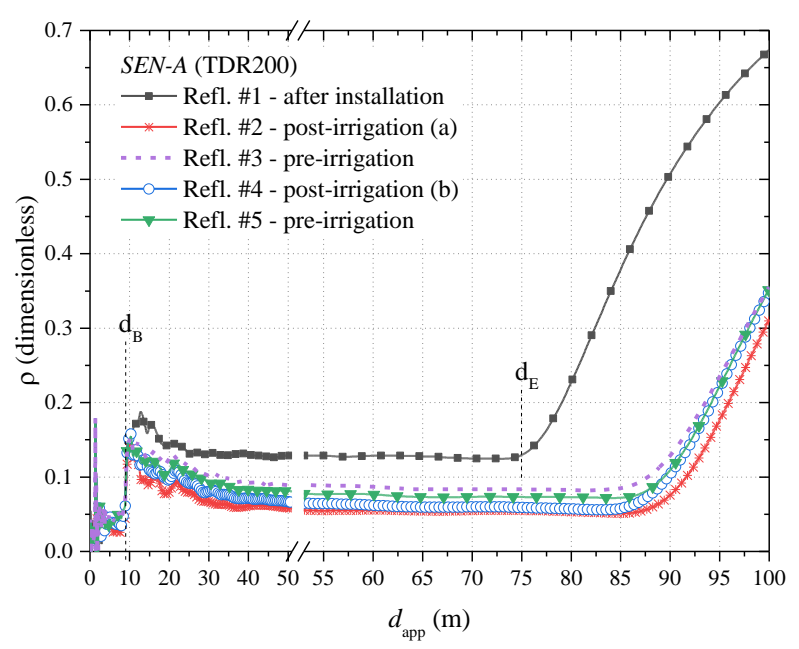

(a)

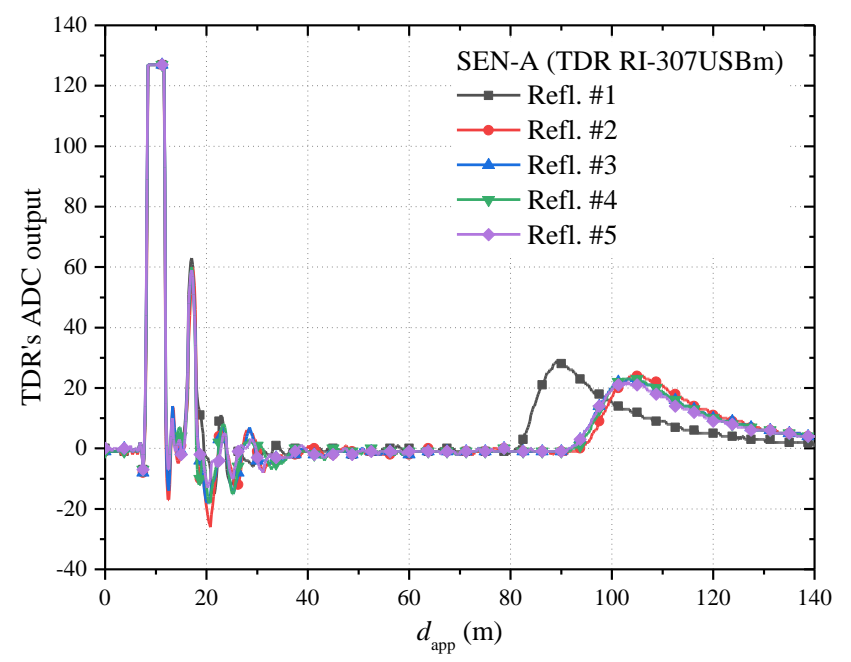

(b)

Figure 4. SEN-A:TDR reflectogram with the TDR200 (a) and with the TDR RI307USBm (b)

- reflectogram \#4: (post-irrigation) acquired approximately 10 hours after the irrigation process was finished,

- reflectogram \#5: pre-irrigation measurement.

Figure 4(a) and 4(b) show the measurements obtained with the TDR200 and TDR RI-307USBm, respectively. It can be seen that after irrigation (reflectogram \#2), the apparent length of the $\mathrm{SE}$ increases, as d-SE shifts towards a longer distance; this is a result of the increased effective permittivity. From reflectogram \#3, acquired after three days and before a new irrigation, it can be seen that the apparent length of the SE has decreased. A similar trend occurs for reflectogram \#4 and reflectogram \#5. Based on the overall length of the SE, the system is able to discriminate well between different soil moisture conditions. This can be used as a parameter for activated irrigation. The TDR200 instrument exhibits better performance in estimating dielectric variations; however, the costs are considerably higher than the TDR RI-307USBm.

In practical applications, the elongated SEs will allow the retrieval of a map in real time that shows the state of the water content in the cultivations, thus allowing an automatic tailormade intervention, especially in view of the optimal management of the irrigation processes. 


\subsection{Experimental results for SEN-B}

For SEN-B, the goal of the proposed method was twofold: 1) an ex-ante monitoring of concrete curing along a building diffuse profile and 2) an ex-post monitoring for the detection of dielectric anomalies as a result of the degradation or stress of the structure. A concrete beam was used as a case study. In order to monitor the water content during the hydration process, three d-SEs were inserted in a concrete beam: one at the bottom of the beam, another in the middle and a third at the top. The hardening phase is considered to be completed within the first 28 days, as after this period more than $90 \%$ of the overall mechanical strength has developed. For this reason, the beam was monitored over the 28-day period by acquiring TDR data for the d-SEs. Figure 5(a) shows some of the TDR reflectograms acquired during the considered period (for clarity of the image, not all the reflectograms are reported); Figure 5(b) shows a zoomed image that highlights the trend.

From the reflectogram, it is possible to identify the beginning and the end of the d-SE (denoted respectively by $d_{B \text {,app }}$ and $d_{E, \text { app }}$ ) in order to calculate the apparent distance $d_{\text {app }}$ of the d$\mathrm{SE}$. As can be seen, this parameter decreases with the hydration

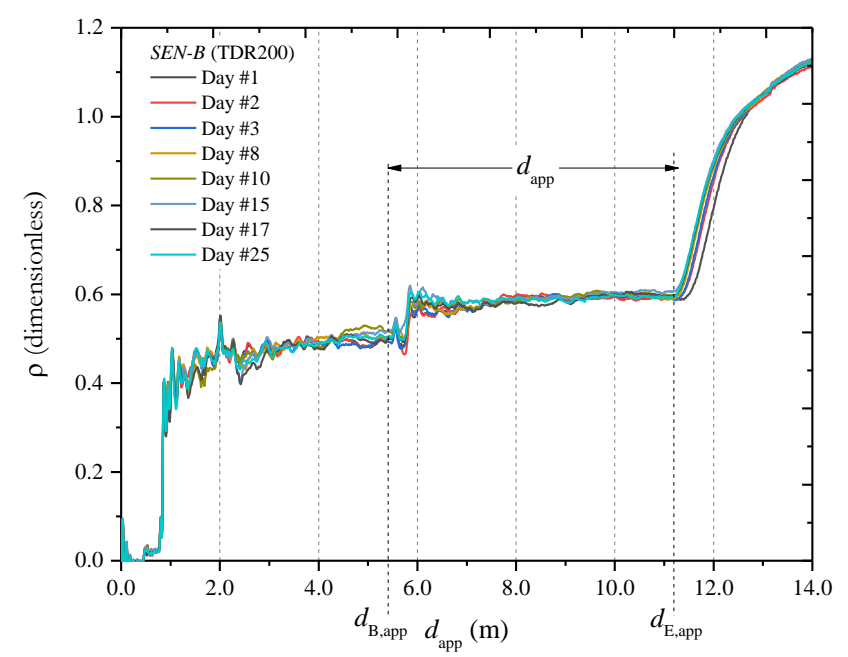

(a)

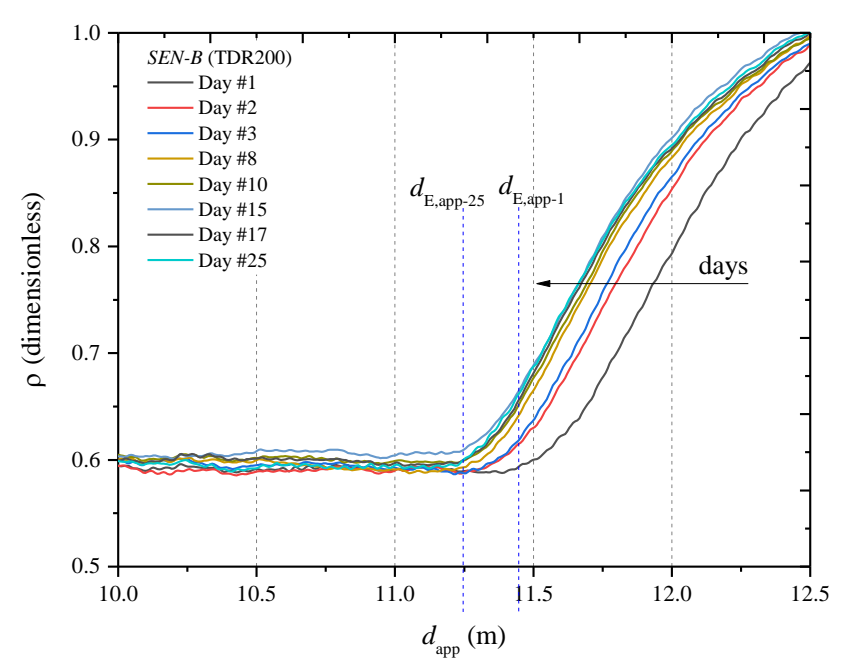

(b)

Figure 5. SEN-B: selected TDR reflectograms for the central d-SE over the 28-day observation period (a) and a zoomed image (b).

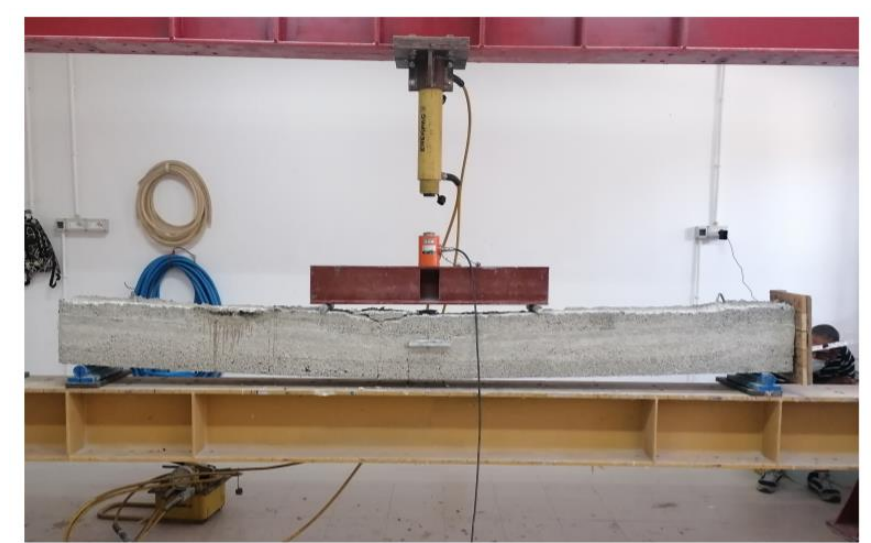

Figure 6. Experimental setup for post-mechanical tests on the beam.

process because $d_{E \text {,app }}$ shifts towards lower values. This indicates that the apparent distance $d_{\text {app }}$ of the d-SE decreases with the decreasing dielectric permittivity $\varepsilon_{\text {app }}$ of the concrete beam as a result of the ongoing hydration process.

However, ex-post monitoring through an embedded low-cost diffused SE can promptly detect the deterioration of the structures. In this regard, mechanical tests on the beam were carried out to analyse whether the diffuse sensor cable was sensitive to mechanical deformation. The beam was subjected to several tests with concentrated weights varying from $1,000 \mathrm{kgf}$ to $9,000 \mathrm{kgf}$, with an increase of 1,000 kgf at each test. Figure 6 illustrates the test setup. As explained in Section 3, the d-SE also included a coaxial cable, which was sensitive to deformation and/or compression phenomena. During the mechanical test, TDR measurements were also carried out on the d-SE.

As is known from the theory, the diameter of the inner and outer conductors determines the impedance of the coaxial cable. Because of this, a deformation in the cable resulting from the degradation phenomena in the structure causes a variation in the electrical impedance, which was immediately identified from the measurements. As shown in Figure 7, as the weight applied to the beam increases, the reflection coefficient decreases, and the apparent distance increases, indicating that the cable is being deformed and bent. These mechanical tests highlight that continuous ex-post monitoring through permanent, d-SEs can provide early indications of structural problems. In this way, safety measures can be considered in time, and structural interventions can be performed immediately.

\section{CONCLUSIONS}

In this study, the development and the proof of concept of a multi-purpose SEN were addressed through the adoption of TDR and d-SEs. The proposed system, which allows the limitations of traditional punctual monitoring systems to be overcome, was validated for the localisation of leaks (SEN-W), for monitoring the diffused profile of the water content of soil in agriculture (SEN-A) and for the ex-ante and ex-post monitoring of the hydration process and the diagnostics of destructive phenomena (SEN-B).

Additionally, the proposed monitoring system can be easily extended to other fields. In practical applications, each SEN could be managed through a portable device and through a single platform. In addition, the monitored data can be stored in a repository and used for further statistics or future reference, and the output of the SENs can be geo-referenced by acquiring the 


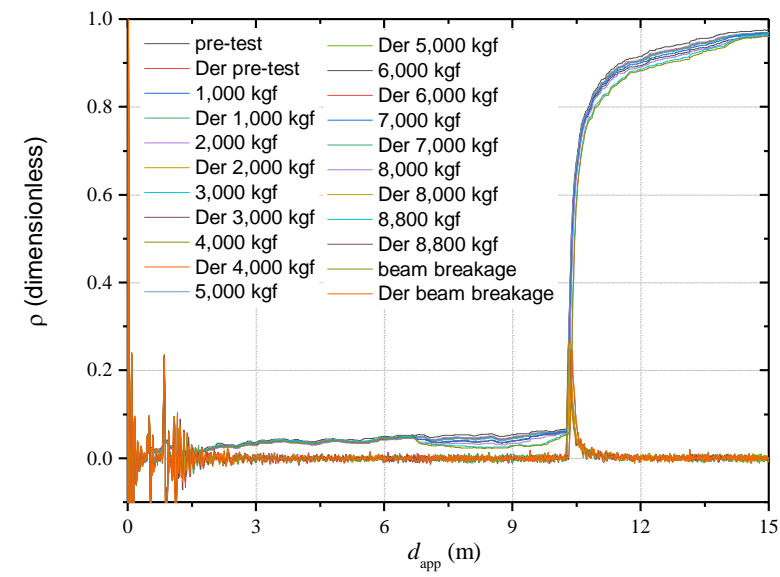

a)

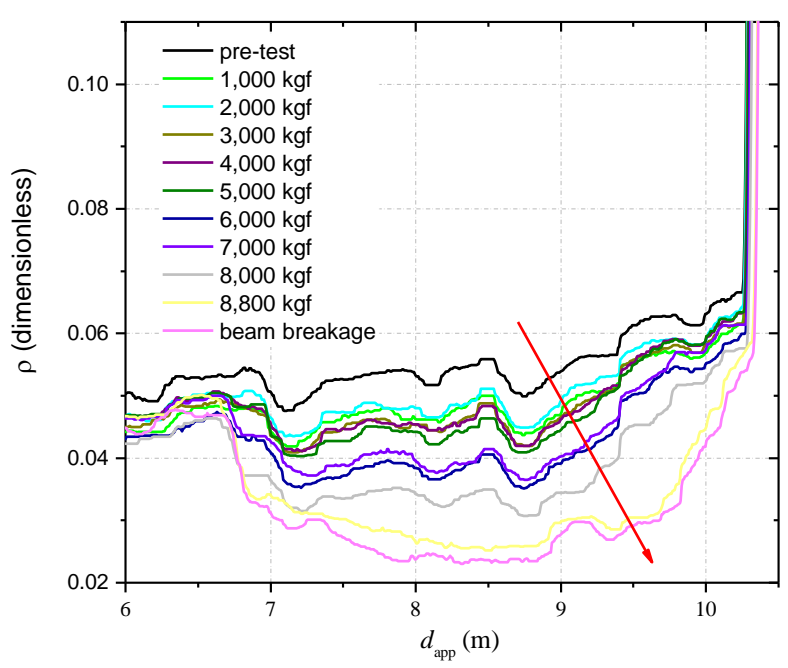

b)

Figure 7. a) TDR reflectograms and first derivatives acquired through the coaxial cable of the d-SE for the concrete beam in the different compression conditions. b) Zoom of the trend as compression increases.

GPS coordinates of the location where the MR measurements are taken. The introduction of these strategies would contribute to making the proposed monitoring system evolve into a cyberphysical measurement system [32], [33], fully exploiting the potential of 4.0 technologies.

\section{REFERENCES}

[1] A. Sforza, C. Sterle, P. D'amore, A. Tedesco, F. De Cillis, R. Setola, Optimization models in a smart tool for the railway infrastructure protection, in: Critical Information Infrastructures Security. E. Luiijf, P. Hartel (editors). Springer, Cham, 2013, pp. 191-196.

DOI: $10.1007 / 978-3-319-03964-0 \quad 17$

[2] P. D'Amore, A. Tedesco, Technologies for the implementation of a security system on rail transportation infrastructures, in: Railway Infrastructure Security. R. Setola, A. Sforza, V. Vittorini, C. Pragliola (editors), Springer, Cham, 2015, ISBN: 978-3-31904425-5, pp. 123-141.

DOI: $10.1007 / 978-3-319-04426-27$

[3] C. Scuro, P. F. Sciammarella, F. Lamonaca, R. S. Olivito, D. L. Carni, IoT for structural health monitoring, IEEE Instrumentation Measurement Magazine 21 (2018) pp. 4-14. DOI: $\underline{\text { 10.1109/MIM.2018.8573586 }}$
[4] E. Sisinni, A. Saifullah, S. Han, U. Jennehag, M. Gidlund, Industrial internet of things: challenges, opportunities, and directions, IEEE Transactions on Industrial Informatics 14 (2018) pp. $4724-4734$. DOI: $10.1109 /$ TII.2018.2852491

[5] B. Chen, J. Wan, L. Shu, P. Li, M. Mukherjee, B. Yin, Smart factory of industry 4.0: key technologies, application case, and challenges, IEEE Access 6 (2018) pp. 6505-6519.

DOI: $10.1109 /$ ACCESS.2017.2783682

[6] H. Xu, W. Yu, D. Griffith, N. Golmie, A survey on industrial internet of things: a cyber-physical systems perspective, IEEE Access 6 (2018) pp. 78238-78259.

DOI: $10.1109 /$ ACCESS.2018.2884906

[7] F. Lamonaca, C. Scuro, D. Grimaldi, R. S. Olivito, P. F. Sciammarella, D. L. Carnì, A layered IoT-based architecture for a distributed structural health monitoring system, Acta IMEKO 8 (2019) 2, pp. 45-52.

DOI: $10.21014 /$ acta imeko.v8i2.640

[8] T. Addabbo, A. Fort, M. Mugnaini, L. Parri, S. Parrino, A. Pozzebon, V. Vignoli, A low power IoT architecture for the monitoring of chemical emissions, Acta IMEKO 8 (2019) 2, pp. 53-61.

DOI: $10.21014 /$ acta imeko.v8i2.642

[9] A. Bernieri, D. Capriglione, L. Ferrigno, M. Laracca, Design of an efficient mobile measurement system for urban pollution monitoring, Acta IMEKO 1 (2012) 1, pp. 77-84. DOI: $10.21014 /$ acta imeko.v1i1.27

[10] G. Y. Chen, X. Wu, E. P. Schartner, S. Shahnia, N. Bourbeau Hébert, L. Yu, X. Liu, A. V. Shahraam, T. P. Newson, H. Ebendorff-Heidepriem, H. Xu, D. G. Lancaster, T. M. Monro, Short-range non-bending fully distributed water/humidity sensors, Journal of Lightwave Technology 37 (2019) pp. 20142022.

DOI: $10.1109 /$ JLT.2019.2897346

[11] L. Zhao, J. Wang, Z. Li, M. Hou, G. Dong, T. Liu, T. Sun, K. T. V. Grattan, Quasi-distributed fiber optic temperature and humidity sensor system for monitoring of grain storage in granaries, IEEE Sensors Journal 20 (2020) pp. 9226-9233. DOI: $10.1109 /$ ISEN.2020.2989163

[12] D.-S. Xu, L.-J. Dong, L. Borana, H.-B. Liu, Early-warning system with quasi-distributed fiber optic sensor networks and cloud computing for soil slopes, IEEE Access 5 (2017) pp. 25437-25444. DOI: 10.1109/ACCESS.2017.2771494

[13] A. Cataldo, E. De Benedetto, G. Cannazza, A. Masciullo, N. Giaquinto, G. D’Aucelli, N. Costantino, A. De Leo, M. Miraglia, Recent advances in the TDR-based leak detection system for pipeline inspection, Measurement 98 (2017) pp. 347-354. DOI: 10.1016/i.measurement.2016.09.017

[14] A. Cataldo, E. De Benedetto, G. Cannazza, N. Giaquinto, M. Savino, F. Adamo, Leak detection through microwave reflectometry: from laboratory to practical implementation, Measurement 47 (2014) pp. 963-970. DOI: $10.1016 /$ i.measurement.2013.09.010

[15] N. Giaquinto, G. D’Aucelli, E. De Benedetto, G. Cannazza, A. Cataldo, E. Piuzzi, A. Masciullo, Criteria for automated estimation of time of flight in TDR analysis, IEEE Transactions on Instrumentation and Measurement 65 (2016) pp. 1215-1224. DOI: $10.1109 /$ TIM.2015.2495721

[16] A. Cataldo, E. De Benedetto, G. Cannazza, E. Piuzzi, N. Giaquinto, Embedded TDR wire-like sensing elements for monitoring applications, Measurement 68 (2015) pp. 236-245. DOI: $10.1016 /$ i.measurement.2015.02.050

[17] A. Cataldo, E. De Benedetto, A. Masciullo, G. Cannazza, A new measurement algorithm for TDR-based localization of large dielectric permittivity variations in long-distance cable systems, Measurement 174 (2021), art. 109066. DOI: $10.1016 /$ i.measurement.2021.109066

[18] A. Walczak, M. Lipiński, G. Janik, Application of the TDR sensor and the parameters of injection irrigation for the estimation of soil evaporation intensity, Sensors 21 (2021) art. 2309. 
DOI: $10.3390 / \mathrm{s} 21072309$

[19] D.-J. Kim, J.-D. Yu, Y.-H. Byun, Horizontally elongated time domain reflectometry system for evaluation of soil moisture distribution, Sensors 20 (2020) pp. 1-17.

DOI: $10.3390 / \mathrm{s} 20236834$

[20] C.-P. Lin, Y. J. Ngui, C.-H. Lin, Multiple reflection analysis of TDR signal for complex dielectric spectroscopy, IEEE Transactions on Instrumentation and Measurement 67 (2018) pp. 2649-2661.

DOI: $10.1109 /$ TIM.2018.2822404

[21] A. Cataldo, E. De Benedetto, G. Cannazza, E. Piuzzi, E. Pittella, TDR-based measurements of water content in construction materials for in-the-field use and calibration, IEEE Transactions on Instrumentation and Measurement 67 (2018) pp. 1230-1237. DOI: $10.1109 /$ TIM.2017.2770778

[22] E. Iaccheri, A. Berardinelli, G. Maggio, T. G. Toschi, L. Ragni, Affordable time-domain reflectometry system for rapid food analysis, IEEE Transactions on Instrumentation and Measurement 70 (2021) pp. 1-7. DOI: $10.1109 /$ TIM.2021.3069050

[23] S. M. Kim, J. H. Sung, W. Park, J. H. Ha, Y. J. Lee, H. B. Kim, Development of a monitoring system for multichannel cables using TDR, IEEE Transactions on Instrumentation and Measurement 63 (2014) pp. 1966-1974. DOI: 10.1109/TIM.2014.2304353

[24] G.-Y. Kim, S.-H. Kang, W. Nah, Novel TDR test method for diagnosis of interconnect failures using automatic test equipment, IEEE Transactions on Instrumentation and Measurement 66 (2017) pp. 2638-2646. DOI: $10.1109 /$ TIM.2017.2712978

[25] C.-K. Lee, S. J. Chang, A method of fault localization within the blind spot using the hybridization between TDR and wavelet transform, IEEE Sensors Journal 21 (2021) pp. 5102-5110. DOI: $10.1109 /$ ISEN.2020.3035754

[26] C. M. Furse, M. Kafal, R. Razzaghi, Y.-J. Shin, Fault diagnosis for electrical systems and power networks: a review, IEEE Sensors Journal 21 (2021) pp. 888-906. DOI: $10.1109 /$ JSEN.2020.2987321
[27] S. Lee, H.-K. Yoon, Hydraulic conductivity of saturated soil medium through time-domain reflectometry, Sensors 20 (2020) pp. 1-18. DOI: $10.3390 / \mathrm{s} 20237001$

[28] A. Cataldo, L. Tarricone, M. Vallone, F. Attivissimo, A. Trotta, Uncertainty estimation in simultaneous measurements of levels and permittivities of liquids using TDR technique, IEEE Transactions on Instrumentation and Measurement 57 (2008) pp. 454-466. DOI: $10.1109 /$ TIM.2007.911700

[29] H. Yang, H. Wen, TDR prediction method for PIM distortion in loose contact coaxial connectors, IEEE Transactions on Instrumentation and Measurement 68 (2019) pp. 4689-4693. DOI: $10.1109 /$ TIM.2019.2900963

[30] G. Robles, M. Shafiq, J. M. Martínez-Tarifa, Multiple partial discharge source localization in power cables through power spectral separation and time-domain reflectometry, IEEE Transactions on Instrumentation and Measurement 68 (2019) pp. 4703-4711 DOI: $10.1109 /$ TIM.2019.2896553

[31] R. Schiavoni, G. Monti, E. Piuzzi, L. Tarricone, A. Tedesco, E. De Benedetto, A. Cataldo, Feasibility of a wearable reflectometric system for sensing skin hydration, Sensors 20 (2020), art. 2833. DOI: $10.3390 / \mathrm{s} 20102833$

[32] S. Grazioso, A. Tedesco, M. Selvaggio, S. Debei, S. Chiodini, E. De Benedetto, G. Di Gironimo, A. Lanzotti, Design of a soft growing robot as a practical example of cyber-physical measurement systems, Proc. of the IEEE Conf. on Metrology for Industry 4.0 and IoT Proceedings, Rome, Italy, 7 - 9 June 2021, pp 23-26.

DOI: $10.1109 /$ MetroInd4.0IoT51437.2021.9488477

[33] S. Grazioso, A. Tedesco, M. Selvaggio, S. Debei, S. Chiodini, Towards the development of a cyber-physical measurement system (CPMS): case study of a bioinspired soft growing robot for remote measurement and monitoring applications, Acta IMEKO 10 (2021) 2, pp. 103-109.

DOI: $10.21014 /$ acta imeko.v10i2.1123 